\title{
Claude Simon dans « la Pléiade »
}

\author{
Alastair B. Duncan
}

\section{(2) OpenEdition}

Journals

Édition électronique

URL : http://journals.openedition.org/ccs/511

DOI $: 10.4000 /$ ccs. 511

ISSN : 2558-782X

\section{Éditeur :}

Presses universitaires de Rennes, Association des lecteurs de Claude Simon

\section{Édition imprimée}

Date de publication : 31 décembre 2006

Pagination : 149-152

ISBN : 9782914518895

ISSN : 1774-9425

\section{Référence électronique}

Alastair B. Duncan, «Claude Simon dans « la Pléiade » ", Cahiers Claude Simon [En ligne], 2 | 2006, mis en ligne le 20 septembre 2017, consulté le 15 septembre 2020. URL : http://journals.openedition.org/ $\operatorname{ccs} / 511$ 


\section{Claude Simon dans « la Pléiade »}

La publication par Gallimard, en mars 2006, d'un volume $d$ 'Euvres de Claude Simon dans la prestigieuse "Bibliothèque de la Pléiade " a été sans aucun doute l'événement majeur de cette année simonienne. Elle a aussi eu un important retentissement médiatique, dans la presse écrite et dans les radios, qui atteste la reconnaissance grandissante de cette ceuvre bien au-delà des cercles intellectuels. Établie par Alastair B. Duncan, avec la collaboration de Jean H. Duffy, cette édition d'une sélection d'ouvrages présente toutes les qualités d'une grande édition critique, à la fois pleine de savoir et d'intelligence, et abordable par un public cultivé : une introduction générale lumineuse, retraçant les grandes phases du développement de l'auvre, des notices sur chaque livre qui sont autant d'études littéraires suggestives, des notes élucidant nombre de références contenues dans le texte simonien (historiques, littéraires, artistiques, géographiques...), des appendices présentant des textes et documents difficilement accessibles qui éclairent le travail de l'écrivain. Ce volume de la Pléiade accompagnera désormais tout lecteur de Claude Simon comme une source inépuisable de plaisir et de réflexion. Alastair B. Duncan a bien voulu en retracer la genèse pour les Cahiers Claude Simon.

Au printemps 2001, Claude Simon a accepté la proposition d'Antoine Gallimard de publier dans la "Bibliothèque de la Pléiade" un choix de ses œuvres en un volume. Le choix des 
ouvrages a été fait par Claude Simon. Il représente toutes les époques de son travail à partir du Vent. Un accent particulier est mis sur les romans de la mémoire restituante : Le Vent (1957), La Route des Flandres (1960), Le Palace (1962). Mais le volume contient aussi, pour la période dite formaliste, La Bataille de Pharsale, œuvre de transition (1969), et Triptyque (1973); et, pour les romans "à base de vécu ", cette grande œuvre récapitulative, Le Jardin des Plantes (1997). En outre, Simon a choisi de mettre en avant le texte publié d'abord en album avec des peintures de Miró et repris plus tard sous le titre La Chevelure de Bérénice (1984). Sur l'incitation de Gallimard, il a ajouté le Discours de Stockholm. Deux autres textes de Simon donnent également le reflet de la conception qu'il se faisait de son ouvre et de son travail et de la façon dont il les situait dans l'histoire littéraire : la préface à Orion aveugle (1970) et " La fiction mot à mot ", communication donnée au colloque de Cerisy sur le Nouveau Roman en 1971. Le volume contient en outre une étude de Mireille Calle-Gruber que Simon prisait beaucoup, "Le récit de la description ou De la nécessaire présence des demoiselles allemandes tenant chacune un oiseau dans les mains ».

Simon m'ayant confié la direction du volume, je lui ai exprimé le désir d'avoir comme collaboratrice ma collègue d'Edimbourg, Jean Duffy, choix que Simon a tout de suite approuvé. Nous nous sommes vite mis d'accord, Jean Duffy et moi, sur les ouvrages dont elle aurait l'entière responsabilité : La Bataille de Pharsale et Le Jardin des Plantes. Mais notre collaboration, et l'aide qu'elle m'a apportée, sont allées beaucoup plus loin. C'est ensemble que nous avons conçu le volume, et notamment le format des notices ; la bibliographie, fruit d'un travail commun, est la version revue et perfectionnée de notre collaboration sur un livre précédent ; et, d'un œil amical et néanmoins impitoyable, Jean a lu et commenté mes brouillons, tout comme j'ai lu les siens. Nous nous sommes ainsi efforcés de donner une certaine homogénéité au volume, le lecteur ne devant pas se trouver dépaysé en passant d'une notice à une autre, même si l'auteur en est différent.

Notre collaboration avec Claude Simon a été un des grands plaisirs de cette Pléiade. La chose n'allait pas de soi. Pour Simon, chaque 
roman était l'aboutissement d'un travail sur la forme; la critique n'avait donc qu'à s'occuper du roman dans sa forme définitive. C'est ainsi que nous n'avons pas eu accès aux manuscrits. Mais les conventions de la Pléiade exigent que l'on parle des origines et de la genèse des œuvres. Très aimablement, Simon a accepté de jouer le jeu : nous avons eu sa permission d'inclure des schémas, des plans et même quelques pages de manuscrits ; et nous avons brièvement évoqué dans chaque notice les quelques éléments de la genèse du roman que nous avons pu recueillir dans des interviews d'époque ou en conversation avec Claude et Réa Simon.

Car c'était dans l'appartement même de Claude Simon que ce travail en partie se faisait. Nous lui avons tous deux envoyé des questions écrites. Au début, en 2001 et en 2002, nous avons parfois reçu des réponses écrites. Mais, de plus en plus fatigué, Simon préférait nous recevoir, séparément, chez lui. Dans la mesure du possible, il répondait à nos questions - nous avons essayé surtout de vérifier des faits et des dates - mais il arrivait aussi qu'il évoque bien d'autres choses ; et les notices et les notes portent le reflet de ces conversations, comme nous avons essayé de le signaler dans le volume. Un dernier aspect de cette collaboration concerne Réa Simon. Quand nos notices et nos notes s'approchaient de leur forme définitive, Réa les a toutes lues et commentées. Elle a corrigé des erreurs factuelles, elle nous a poussés, avec une grande finesse, vers plus de clarté et de précision et elle a veillé à ce que la différence soit toujours faite entre nos vues propres et la perspective de Simon qu'il ne fallait pas trahir.

Enfin, dans ce volume des Cahiers consacré à la mémoire de Claude Simon, Jean Duffy voudrait se joindre à moi pour rendre hommage à l'écrivain tel que nous l'avons connu. Je suis allé pour la première fois chez lui à Paris en 1967 ou en 1968, et Jean quelques années plus tard en 1979. À chaque fois, son accueil était le même : simple, chaleureux, attentif, toujours prêt à vous sortir une photo, une carte postale, la lettre manuscrite d'un ancêtre. Nous lui envoyions parfois nos travaux qu'il lisait en anglais aussi bien qu'en français et qu'il commentait en toute franchise. C'était un homme passionné et d'une grande rectitude : ses jugements étaient sans appel et ses enthousiasmes - pour l'Angleterre, Churchill, le rugby 
- débordants. Son humour, dont on n'a pas suffisamment parlé, allait de pair avec une grande et étonnante modestie. Tout critique n'a pas la bonne fortune de travailler sur un écrivain dont la personne est aussi attachante que l'œuvre. Nous avons eu le grand privilège de travailler sur Claude Simon; il était impossible de ne pas l'aimer.

\section{Alastair B. DUNCAN}

\section{Manifestations}

Le "Séminaire Claude Simon ", animé par Dominique Viart et Didier Alexandre, s'est poursuivi dans les locaux de l'École Normale Supérieure, rue d'Ulm (Paris, $\left.5^{\mathrm{e}}\right)$ :

- 6e journée (samedi 11 juin 2005). «Simon : style, syntaxe ». Interventions de Pascal MOUGIN ("Claude Simon : phrase et habitus "), Ilias YOCARIS ("Une poétique de l'indétermination : style et syntaxe dans La Route des Flandres", David ZEMMOUR ("La syntaxe de Claude Simon ou "l'ordre sensible des choses" »), Marie-Albane RIOUX-WATINE ("Le dialogue simonien : enjeux, formes, déviances »).

- $7^{e}$ journée (samedi 28 janvier 2006). "Simon et l'existentialisme". Interventions de Jean-François LOUETTE ("Claude Simon et Sartre dans les premiers romans »), Didier ALEXANDRE ("Claude Simon et Sartre dans le discours critique », Dominique VIART ("Littérature engagée et fiction critique : de Sartre à Simon »).

- 8 e journée (samedi 10 juin 2006). «Lectures de L'Herbe». Interventions de Gérard ROUBICHOU (" (Re)lire L'Herbe en $2006 »)$, Alexey VISHNYAKOV ("Lecture de L'Herbe à travers ses unités de seuil - titre, épigraphe, incipit, explicit»), Anne-Yvonne JULIEN ("Le dire du "Rien" dans L'Herbe. Versions simoniennes du Nihil»), Michel BERTRAND ("Inscriptions de l'Histoire dans L'Herbe de Claude Simon »).

Les prochaines journées auront lieu le samedi 3 février 2007 (« Claude Simon et Georges Bataille ») et le samedi 9 juin 2007 ("Lectures du Vent»). Le programme détaillé sera envoyé aux adhérents et accessible dans les pages internet de l'Association, hébergées par Christine Genin sur son site Labyrinthe (adresse : http://perso.orange.fr/labyrinthe/simonasso.htm). 